\title{
Heat Transfer Mechanisms in Nanofluids: A Review
}

\author{
Gaurab Kr. Ghosh \\ Department of Mechanical Engineering, Indian School of Mines, Dhanbad, Jharkhand, 826004, India
}

\begin{abstract}
Traditional heat transfer fluids, such as water, ethylene glycol and lubricating oil which are used in various applications such as chemical processes, refrigeration, heating and cooling processes, transportation, power generation and other micro-sized applications have poor heat transfer properties and impose limitation to heat transfer augmentation. Improving the thermal conductivity is the key idea to improve the heat transfer characteristics of conventional fluids. Since a solid matrix has a larger thermal conductivity relative to base fluid, suspending solid fine particles (millimeter or micrometer sized range) into the base fluid is expected to improve the thermal conductivity. A dilute suspension of nanometer-sized particles dispersed in a base fluid is known as Nanofluids. Nanofluids exhibit enhanced heat transfer properties and are expected to be a promising coolant candidate for thermal management systems of next generation.
\end{abstract}

Keywords: Nanofluids, Heat transfer enhancement, Thermophysical properties

\section{Introduction}

Over a century ago, researchers have tried to increase the thermal conductivity of suspensions by adding solid fine particles but those techniques are discarded due to associated drawbacks with them. However, the recent advances in nanotechnology have made it possible to produce particles with nano-sized order (nanoparticles) with relative ease. Innovative idea of suspending these nanoparticles in a base fluid for improving thermal conductivity has been proposed recently [1-3]. These new class of fluids are called 'nanofluids'. Nanofluids were introduced in 1995 [4] by Choi in Argonne National Laboratory (ANL). Due to their small size, nanoparticles can easily fluidize inside the base fluid, and as a result, the clogging of channels and erosion in channel walls are no longer a problem. Compared with suspended particles of millimeter or micrometer dimensions, nanofluids show not only better suspension stability and rheological properties, but also higher thermal conductivity. Earlier studies have shown that there is about $40 \%$ enhancement in thermal conductivity of nano-fluids is possible with 0.3 vol. \% of copper nanoparticles of about 10 nanometer size [5]. This shows thermal conductivity enhancement at nano-scales not only volume but also size dependent. The ratio of surface area to volume is 1000 times greater for particles with a $10 \mathrm{~nm}$ diameter than that for particles with $10 \mu \mathrm{m}$ diameter. The much larger surface area of nanoparticles relative to those of conventional particles should not only improve heat transfer capabilities, but also increase the stability of suspensions. Nanofluids offer a unique combination of the two features most highly desired for thermal engineering applications: good stability against sedimentations and ultra-high thermal conductivity. By suspending nanophase particles in heating or cooling fluids, the heat transport performance of the fluid can be significantly improved [2]. The main reasons may be listed as follows:

1) The suspended nanoparticles increase the surface area and the heat capacity of the fluid.

2) The suspended nanoparticles increase the effective (or apparent) thermal conductivity of the fluid.

3) The interaction and collision among particles, the surface of fluid and the flow passage are intensified.
4) The dispersion of nanoparticles flattens the transverse temperature gradient of the fluid.

\section{Nanofluid Synthesis Techniques}

Many different particle materials are used for nanofluid preparation. $\mathrm{Al}_{2} \mathrm{O}_{3}, \mathrm{CuO}, \mathrm{TiO}_{2}, \mathrm{SiC}, \mathrm{TiC}, \mathrm{Ag}, \mathrm{Au}, \mathrm{Cu}$, and $\mathrm{Fe}$ nanoparticles are frequently used in research. However, these particles show different thermal conductivity enhancement and are not related anyway. Spherical particles are mostly used in nanofluids for the simplicity of analysis.

\section{One Step Method}

Preparation of nanofluids is the first key step in the application of nanofluid cooling technology. In the one-step technique, the production of nanoparticles and dispersion of nanoparticles in the base fluid are combined into a single step. There are some variations of this technique. In one of the common methods, named direct evaporation one step method, the nanofluid is produced by the solidification of the nanoparticles, which are initially gas phase, inside the base fluid [5]. The dispersion characteristics of nanofluids produced with one-step method are better than those produced with the two-step technique [6]. The main drawback of this technique is that they are not proper for mass production, which limits their commercialization [6].

\section{Two Step Method}

In the two-step technique, the first step is the production of nanoparticles and the second step is the dispersion of the nanoparticles in a base fluid. Two-step technique is advantageous when mass production of nanofluids is considered, because at present, nanoparticles can be produced in large quantities by utilizing the technique of inert gas condensation [7]. The main disadvantage of the two-step technique is that the nanoparticles cluster during the preparation of the nanofluid which prevents the proper dispersion of nanoparticles inside the base fluid. [6].

\section{Enhancement Mechanisms and Associated Models}

Different authors proposed different mechanisms of heat transfer in nanofluids [19-22]. The proposed mechanisms for 


\section{International Journal of Science and Research (IJSR) \\ ISSN (Online): 2319-7064}

Index Copernicus Value (2013): 6.14 | Impact Factor (2015): 6.391

the anomalous enhancement discussed in the open literature are:

\section{Brownian Motion of Nanoparticles}

Unlike larger sized particles, nanoparticles are not static in solution. As particle dimensions get smaller and smaller (particularly of nano-sized order), it becomes increasingly difficult to hold them in place. They have a natural tendency to move around and hence Brownian diffusion or motion becomes a major effect. Brownian motion is the random motion of particles suspended in a fluid at very high rates. This random motion transports energy directly by nanoparticles. The energy exchange in the direct nanoparticle-nanoparticle contact arising from the particle collisions in the nanofluid could result in an enhancement of the thermal conductivity. Brownian motion is a very small fraction of the thermal conductivity of the base fluid. This fact was verified by molecular dynamics simulation [33]. As a result, it was concluded that Brownian motion of nanoparticles could not be the main cause of anomalous thermal conductivity enhancement with nanofluids. Many models were developed for the determination of thermal conductivity of nanofluids based on the Brownian motion of nanoparticles. Jang and Choi [24] modelled the thermal conductivity of nanofluids by considering the effect of random motion i.e. Brownian motion of nanoparticles. The proposed model is a function of not only thermal conductivity of the base fluid and nanoparticles but it also depends on the temperature and size of nanoparticles. Energy transport in nanofluids was considered of four modes; heat conduction in the base fluid, heat conduction in nanoparticles, collision between nanoparticles (due to Brownian motion) and micro-convection caused by the random motion of the nanoparticles. Among these, the collisions between nanoparticles were found to be negligible when compared to other modes. As a result of the consideration, the following expression was presented:

$$
k_{e f f}=k_{f}(1-\phi)+k_{p}^{*} \phi+3 C_{1} \frac{d_{f}}{d p} k_{f} \operatorname{Re}_{d}{ }^{2} \operatorname{Pr}_{f} \phi
$$

Where $C_{1}$ is proportionality constant, $d_{f}$ is the diameter of fluid molecules, $\operatorname{Pr}_{f}$ is the prandtl number of base fluid and $k_{p}{ }^{*}$ is defined so that it also includes the effect of Kapitza resistance. Koo and Kleinstreuer [12] considered the effect of of thermal conductivity enhancement due to both Brownian motion and static contribution on effective thermal conductivity of nanofluids. This model takes into account the effect of particle dynamics. For the calculation of thermal conductivity of static part ( $\left.k_{\text {static }}\right)$, Maxwell's model is

used (10). For contribution of Brownian motion of particles, $k_{\text {brownian }}$ was considered together with the effect of fluid particles moving with nanoparticles around them. As a result, the following expression was proposed.

$$
k_{\text {brownian }}=5 \times 10^{4} \gamma \phi \rho_{m} C_{p, m} \sqrt{\frac{k_{B} T}{\rho_{p} d_{p}}} f
$$

In this analysis, the interactions between nanoparticles and fluid volumes moving around them are not considered and an additional term $\gamma$ was introduced to take that effect into account. It was indicated that this term becomes more effective with increasing volume fraction. Another parameter $f$ was introduced to model in order to increase the temperature dependency of the model. Both $f$ and $\gamma$ were determined by utilizing the available experimental data. It is difficult to determine the theoretical expressions for $f$ and $\gamma$ due to complexities involved and this can be considered as a drawback of the model.

\section{Clustering of Nanoparticles}

Nanoparticles are known to form clusters [20]. Clustering is the formation of larger particles through aggregation of nanoparticles. If particles cluster into percolating networks, they would create higher conductive paths and thereby have a major effect on the effective thermal conductivity. Evans et al. [21] proposed that clustering can result in fast transport of heat along relatively large distances since heat can be conducted much faster by solid particles when compared to liquid matrix. However, severe clustering of nanoparticles is undesired as this may lead to sedimentation, which decreases thermal conductivity enhancement. Xuan et al. [13] studied the thermal conductivity of nanofluids by considering Brownian motion and clustering of nanoparticles. He proposed an equation to predict the thermal conductivity of nanofluids:

$$
\frac{k_{e f f}}{k_{m}}=\frac{k_{p}+2 k_{m}-2 \phi\left(k_{m}-k_{p}\right)}{k_{p}+2 k_{m}+\phi\left(k_{m}-k_{p}\right)}+\frac{\rho_{p} \phi C_{P, p}}{2 k_{m}} \sqrt{\frac{k_{B} T}{3 \pi r_{c l} \mu_{m}}}
$$

$r_{c l}$ is the apparent radius of the nanoparticle clusters, which should be determined by experiment. The first term on the right hand side of equation (3) is the Maxwell model for thermal conductivity of suspensions of solid particles in fluids. The second term on the right side of above equation adds the effect of random motion of nanoparticles into account. For the contribution of this term, the following values were presented for $\mathrm{Cu}(50 \mathrm{~nm}) /$ water nanofluid: For $\phi=0.03 \%$, contribution of the second term is $11 \%$ when clustering occurs and $17 \%$ when clustering does not occur. For $\phi=0.04 \%$, contribution of the second term is $14 \%$ when clustering occurs and $24 \%$ when clustering does not occur. It was indicated that Brownian motion of nanoparticles become more effective with increasing temperature. On the other hand, as nanoparticles (or clusters) become larger, their random motion becomes slower and this decreases the enhancement in thermal conductivity. It is concluded that severe clustering had adverse effect on thermal conductivity enhancement.

\section{Interfacial Layer Effect around Nanoparticles}

Liquid molecules are known to form ordered layered structures (shells or solid like liquid layer) at solid surfaces and these interfacial layers have different thermophysical properties from the bulk liquid and solid particles. Because of the ordered structure of the nanolayer, it is expected to have higher thermal conductivity than the bulk liquid [22]. The thickness of this solid like layer of liquid molecules at the interface of particles play an important role in heat transportation from solid to adjacent liquid. For particles of micro-meter size, the surface areas are small, e.g., for 


\section{International Journal of Science and Research (IJSR) \\ ISSN (Online): 2319-7064}

Index Copernicus Value (2013): 6.14 | Impact Factor (2015): 6.391

alumina powders with an average diameter of $10 \mu \mathrm{m}$, their SSA (Specific Surface Area) is only $0.15 \mathrm{~m}^{2} \mathrm{~g}^{-1}$. Therefore the effect of interfacial layer is negligible. However, nanoparticles have large SSA, e.g., for alumina powders with an average diameter of $10 \mathrm{~nm}$, their SSA is as large as $151 \mathrm{~m}^{2} \mathrm{~g}^{-1}$ which is much larger compared to micro-sized particle. Thus, the interfacial layer effect around nanoparticle plays a significant role in heat transfer between nanoparticles and base fluid. Various authors have proposed the mathematical models for finding effective thermal conductivity of nanofluid considering interfacial layer formed on particles. Yu \& Choi [25] developed a model for the thermal conductivity of non aggregated particles in nanofluid considering the effect interfacial layer around the nanoparticles, and is expressed as:

$$
k_{e f f}=\frac{k_{p e}+2 k_{m}+2\left(k_{p e}-k_{m}\right)(1+\delta)^{3} \phi}{k_{p e}+2 k_{m}-\left(k_{p e}-k_{m}\right)(1+\delta)^{3} \phi} k_{m}
$$

Based on effective medium theory, the equivalent thermal conductivity $\left(k_{p e}\right)$ of the equivalent particles can be calculated as [25]:

$k_{p e}=\frac{\left[2\left(r_{p}\right)^{3}\left(k_{p}-k_{l}\right)+\left(r_{p}+t\right)^{3}\left(k_{p}+2 k_{l}\right)\right] k_{l}}{k_{l}-k_{p}+\left(r_{p}+t\right)^{3}\left(k_{p}+2 k_{l}\right)}$

And,

$$
\delta=\frac{t}{r_{p}}
$$

Xie et al. [29] proposed a model for the effective thermal conductivity of nanofluid considering the effect of interfacial nanolayer and expressed as:

$$
k_{\text {eff }}=k_{m}+3 \theta \phi_{e} k_{m}+\frac{3 \theta^{2} \phi_{e}^{2}}{1-\theta \phi_{e}} k_{m}
$$

Where,

$$
\theta=\frac{\left(\frac{k_{l}-k_{m}}{k_{l}+2 k_{m}}\right)\left[(1+\delta)^{3}-\frac{\left(\frac{k_{p}-k_{l}}{k_{p}+2 k_{l}}\right)}{\left.\left(\frac{k_{m}-k_{l}}{k_{m}+2 k_{l}}\right)\right]}\right.}{(1+\delta)^{3}+2\left(\frac{k_{l}-k_{m}}{k_{l}+2 k_{m}}\right)\left(\frac{k_{p}-k_{l}}{k_{p}+2 k_{l}}\right)}, \phi_{e}=\phi(1+\delta)^{3}
$$

and,

$$
k_{l}=\frac{k_{m}\left[\frac{k_{p}}{k_{m}}(1+\delta)-1\right]^{2}}{\left\{\left[\frac{k_{p}}{k_{m}}(1+\delta)-1\right]-\delta\right\} \ln \left\{1+\left[\frac{k_{p}}{k_{m}}(1+\delta)-1\right]\right\}+\delta\left[\frac{k_{p}}{k_{m}}(1+\delta)-1\right]}
$$

Mintsa et. al. [30] derived an empirical relation on the basis of experimental data as:

$$
k_{\text {eff }}=[1.72 \phi+1.0] k_{m}
$$

Feng et al. [31] proposed a one-dimensional heat conduction model comprising equivalent particle $\left(r_{p}+t\right)$ and used the thermal-electrical analogy technique to evaluate the effective thermal conductivity for the cluster as:

$$
k_{\text {agg }}=\left[\left(1-\frac{3}{2} \phi_{e}\right) k_{m}+\frac{3 k_{m} \phi_{e}}{\beta}\left[\frac{1}{\beta} \ln \frac{1}{1-\beta}-1\right]\right]
$$

Where, $\beta=1-\frac{k_{m}}{k_{p e}}$

The effective thermal conductivity of nanofluid is a function of thermal conductivity of interfacial nanolayer. It is also fact that some nanoparticles in nanofluid are in contact with each other forming clusters. Feng et al. [31] proposed a model for combined effect of volumetric proportion of aggregate and non aggregate particles as:

$$
k_{\text {eff }}=\phi_{e} k_{\text {agg }}+\left(1-\phi_{e}\right) k_{\text {non-agg }}
$$

The above equation (9) shows that the effective thermal conductivity depends upon the effective volume fraction, thermal conductivity of aggregate and non-aggregate particles. In the above equation the values of effective conductivities of non aggregate and aggregate nanofluid, has been taken from equations (4) and (8) respectively.

\section{Ballistic Phonon Transport in Nanoparticles}

Macroscopic theories assume that the heat is transported by diffusion. In crystalline solids, heat is carried by phonons, that is, by the propagation of lattice vibration. Such phonons are created at random, propagate in random directions and are scattered by each other or by defects. When the size of the nanoparticles in a nanofluid becomes less than the phonon mean- free path, phonons are no longer diffuse across the nanoparticle but move ballistically without any scattering [23]. Therefore, the ballistic phonons initiated in one particle can persist in the liquid and reach a nearby particle even though the phonon mean free path in liquid is very short $(\sim 1-2 \mathrm{~nm})$. This process can further largely by agitated by the random motion of nanoparticles. Therefore, this particular thermal transport process can increase the thermal conductivity to a larger extent.

\section{Thermophysical Properties}

Over the years, a significant amount of experimental and theoretical research was made to investigate the thermophysical behaviour of nanofluids. In the studies, it was observed that a high thermal conductivity enhancement could be obtained with nanofluids. Many researchers proposed theoretical models to explain and predict that anomalous behaviour of thermal conductivity.

\section{Thermal Conductivity}

There are no theoretical formulas currently available to predict the effective thermal conductivity of nanofluids satisfactorily. A variety of theoretical models have been developed to predict the effective thermal conductivity of nanofluids. Maxwell [10] proposed a model for effective thermal conductivity $\left(k_{e f f}\right)$ of suspension of solid particles ( $k_{p}$ ) in the based fluid of conductivity $\left(k_{m}\right)$ for low volume fraction $(\phi)$ of spherical particles and is given by:

$$
k_{e f f}=\frac{k_{p}+2 k_{m}+2 \phi\left(k_{p}-k_{m}\right)}{k_{p}+2 k_{m}-\phi\left(k_{p}-k_{m}\right)} k_{m}
$$




\section{International Journal of Science and Research (IJSR) \\ ISSN (Online): 2319-7064}

Index Copernicus Value (2013): 6.14 | Impact Factor (2015): 6.391

Hamilton Crosser [15] proposed a model with considering the effect of shape of the solid particles in base fluid resulting in:

$$
k_{e f f}=\frac{k_{p}+(n-1) k_{m}-(n-1) \phi\left(k_{m}-k_{p}\right)}{k_{p}+(n-1) k_{m}+\phi\left(k_{m}-k_{p}\right)},
$$

Where, empirical shape factor and is defined as $n=\frac{3}{\psi}$, where $\psi=$ sphericity

Jeffrey [5] modified the Maxwell's equation by taking into account the effects of particle-particle interaction:

$$
\frac{k_{e f f}}{k_{m}}=1+3 \beta \phi+\left(3 \beta^{2}+\frac{3 \beta^{3}}{4}+\frac{9 \beta^{3}}{16} \frac{\alpha+2}{2 \alpha+3}+\frac{3 \beta^{4}}{64}+\ldots \ldots\right) \phi^{2}
$$

Where $\alpha$ is the thermal conductivity ratio, $\frac{K_{e f f}}{K_{m}}$ and $\beta$ is defined as

$\beta=\frac{\alpha-1}{\alpha+2}$. Equation (12) is accurate up to order of $\phi^{2}$.The higher-order terms represent pair interactions of randomly dispersed spheres.

\section{Viscosity}

Drew and Passman [14] suggested the well-known Einstein's equation [20] for calculating viscosity, which is applicable to spherical particle in volume fraction less than 5.0 vol. $\%$ and is defined as follows:

$$
\mu_{n f}=\mu_{b f}(1+2.5 \phi)
$$

Batchelor [16] modified this equation for spherical particles as follows:

$$
\mu_{n f}=\mu_{b f}\left(1+2.5 \phi+6.2 \phi^{2}\right)
$$

Brinkman [17] extended the Einstein's equation for volume fraction less than $4 \%$ as:

$$
\mu_{n f}=\mu_{b f} \frac{1}{(1-\phi)^{2.5}}
$$

Where $\mu_{\mathrm{nf}}$ is the viscosity of the nanofluid and $\mu_{\mathrm{bf}}$ is the viscosity of the base fluid.

\section{Density and Heat Capacity}

The correlation suggested by Pak and Choi [8] is found to be an appropriate way to estimate the density as well as heat capacity necessary to simulate the flow characteristics of a nanofluid. These thermophysical properties are expressed as weighted average of base fluid and nanoparticle properties. In most computational studies, these relations are used which are indicated in terms of particle volume fraction:

$$
\begin{aligned}
& \rho_{n f}=(1-\phi) \rho_{b f}+\phi \rho_{p} \\
& C_{P}=(1-\phi) C_{P, b f}+\phi C_{P, p}
\end{aligned}
$$

\section{Applications}

The novel and advanced concepts of nanofluids offer fascinating applications over a wide range from automotive cooling to biomedical field. Their practical applications are yet to be explored to the fullest once the science and engineering of nanofluids are fully understood. Some of the research work under the application of nanofluid is enlisted below:

Leong et al. [32] studied the application of ethylene glycol based copper nanofluids in an automotive cooling system. They observed that overall heat transfer coefficient \& heat transfer rate in engine cooling system increased with the usage of nanofluids compared to ethylene glycol alone. They showed that about $3.8 \%$ of heat transfer enhancement could be achieved with the addition of $2 \%$ copper particle in a base fluid at Reynolds number 6000 and 5000 for air \& coolant respectively. Nanofluids can also be effectively used for a wide variety of industries, ranging from transportation to energy production and in electronics systems like microprocessors, Micro-Electro-Mechanical Systems (MEMS) and in the field of biotechnology. A nanofluid coolant could flow through tiny passages in MEMS to improve its efficiency [35].

Applications of nanofluid in medicine and biology are also fast growing. One such application is laser surgery. Pulsed lasers are used extensively in laser surgery as nanoblades. A tightly focused laser beam is well capable of inducing hot localized plasma for rapid heating to destroy the tumor cells. Hot local spots will indiscriminately ruins healthy and cancerous cells. Efficient and fast heat dissipation is critical to save the healthy cells in close proximity to targeted tumor cells. Creating a nanofluid environment surrounding the tumor cells will effectively act as a heat sink to preserve the health tissues [34].

Nanofluids are promising and efficient fluids from thermal performance viewpoint. Research about nanofluid is fast growing and the challenges in the production of nanofluids are to develop new methods to cost-effective mass production of nanofluids at an industrial-scale for commercial applications. In ideal case, a method that does not need vacuum system and show capabilities to provide continuous fluid feed and nanofluid extraction is the potential candidate.

\section{Concluding Remarks and Future Scope}

The present review provides a comprehensive outline of various potential heat transfer enhancement mechanisms in nanofluids. Considerable research progress in the field of nanofluids has been pointed out. This review aims to provide an overview of various research work undertaken in the regime of heat transfer enhancement in nanofluids.

At present, there is significant discrepancy in thermal conductivity data of nanofluids. For practical application of nanofluids as 'cooling fluids', these discrepancies should be minimised or eliminated if possible by systematically investigating the effects of various parameters on the thermal conductivity. These parameters could include the effect of agglomeration, $\mathrm{pH}$ value of the suspension, non-dispersion of these nanoparticles in the base fluids etc. The characterization of these nanoparticles should be properly understood to make use of nanofluids in real scenario.

\section{Volume 5 Issue 6, June 2016 www.ijsr.net}




\section{International Journal of Science and Research (IJSR) \\ ISSN (Online): 2319-7064}

Index Copernicus Value (2013): 6.14 | Impact Factor (2015): 6.391

In the literature, there are many models to predict the effective thermal conductivity of nanofluids but there is no single model which determines the effective thermal conductivity of nanofluids taking all possible mechanisms of heat transfer enhancement into account. The concept of keeping 'well dispersed' nanoparticles in the suspension by using acoustic field can be thought of as a future work in this regard. Also some work in 'nano-particle characterization' needs to be carried out to precisely control these particles in various heat transfer applications.

\section{References}

[1] Anna S. Cherkasova, Jerry W. Shan, Particle AspectRatio effects on the thermal conductivity of micro- and nanoparticle suspensions, Journal of Heat Transfer ASME Vol.130 (2008).

[2] Y. Xuan, Q. Li, Heat transfer enhancement of nanofluids, International Journal of Heat and Fluid Flow 21 (2000): 58-64.

[3] Y. Xuan, W. Roetzel, Conceptions for heat transfer correlations of nanofluids, Int. J. Heat Mass Trans. 43 (2000) 3701-3707.

[4] S.U.S. Choi, Enhancing Thermal Conductivity of Fluids with Nanoparticles, ASME FED 231 (1995)99-103.D.

[5] J. A. Eastman, S. U. S. Choi, S. Li, W. Yu, L.J. Thompson, Anomalously increased effective thermal conductivities of ethylene glycol-based nanofluids containing copper nanoparticles, Applied Physics Letters 78 (2001) 718-720.

[6] W. Yu, D. M. France, J. L. Routbort, S. U. S. Choi, S. U. S., Review and Comparison of Nanofluid Thermal Conductivity and Heat Transfer Enhancements, Heat Transfer Eng., 29(5) (2008), pp. 432-460.

[7] J. M. Romano, J. C. Parker, Q. B. Ford, Application Opportunities for Nanoparticles Made from the Condensation of Physical Vapors, Adv. Pm. Part.,(1997) pp. 12-13.130.

[8] B. C. Pak, Y. I Cho, Hydrodynamic and Heat Transfer Study of Dispersed Fluids with Submicron Metallic Oxide Particles, Exp. Heat Transfer, 11(2) (1998), pp. 151-170.

[9] K.S. Hwang, S. P. Jang, S. U. S. Choi, Flow and Convective Heat Transfer Characteristics of WaterBased $\mathrm{Al}_{2} \mathrm{O}_{3}$ Nanofluids in Fully Developed Laminar Flow Regime, Int. J. Heat Mass Tran., 52(1-2) (2009), pp. 193-199.

[10] J. C. Maxwell, A Treatise on Electricity and Magnetism, Clarendon Press, Oxford 1873

[11]H. Xie, J. Wang, T. Xi, Y. Liu, F. Ai, Q. Wu,(2002) Thermal conductivity enhancement of suspensions containing nanosized alumina particles, Journal of Applied Physics 91(7) (2002) 4568-4571.

[12] J. Koo, C. Kleinstreuer, A new thermal conductivity model for nanofluids, J.Nanopart. Res., 6(6) (2004), pp. 577-588.

[13] Y. Xuan, Q. Li, W. Hu, Aggregation Structure and and Thermal Conductivity of Nanofluids, AICHE Journal, 49 (4) (2003), pp.1038-1043.

[14]D. A. Drew, S. L. Passman, Theory of multicomponent fluids, Springer, Berlin, (1999), pp. 121-128.
[15] R.L. Hamilton, O.K. Crosser, Thermal conductivity of heterogeneous two component system, Industrial and Engineering Chemistry 1(3) (1962) 187-191.

[16] G. K. Batchelor, The effect of Brownian motion on the bulk stree in a suspension of spherical particles. Journal of Fluid Mechanics 83 (1977):97-117.

[17]H. C. Brinkman, The Viscosity of Concentrated Suspensions and Solutions, J. Chem. Phys., 20(4) (1952), p. 571.

[18] J. D Anderson, a book of Computational Fluid Dynamics, page no. 15-59.

[19] S.P. Jang, S.U.S. Choi, Role of Brownian motion in the enhanced thermal conductivity of nanofluids, Applied Physics Letters 84 (2004) 4316..

[20] R. Prasher, P. E. Phelan, P. Bhattacharya, 2006, "Effect of Aggregation Kinetics on the Thermal Conductivity of Nanoscale Colloidal Solutions (Nanofluid)," Nano Lett., 6(7) (2006), pp. 1529-1534.

[21] W. Evans, R. Prasher, J. Fish, P. Meakin, P. Phelan, P. Keblinski, Effect of Aggregation and Interfacial Thermal Resistance on Thermal Conductivity of Nanocomposites and Colloidal Nanofluids, Int. J. Heat Mass Tran., 51(5-6) (2008), pp. 1431-1438.

[22] C.W. Nan, G. Liu, Y. Lin, M. Li, Interface effect on thermal conductivity of carbon nanotube composites, Applied Physics Letters 85 (2004) 3549.

[23]P. Keblinski, S.R. Phillpat, S.U.S. Choi, J.A. Eastman, Mechanisms of heat flow in suspension of nano-sized particles (nanofluids), Int. Journal of Heat and Mass Transfer 45(2002) 855-865.

[24] S. P. Jang, S. U. S. Choi, Role of Brownian Motion in the Enhanced Thermal Conductivity of Nanofluids, Appl. Phys. Lett., 84(21) (2004), pp. 4316-4318

[25] W. Yu, S.U.S. Choi, The role of interfacial layers in the enhanced thermal conductivity of nanofluids: A renovated Maxwell model, Journal of Nanoparticle Research 5 (2003) 167-171.

[26] K.C. Leong, C. Yang, S.M.S Murshed, A model for the thermal conductivity of nanofluid- the effect of interfacial layer, Journal of Nanoparticle Research 8 (2006) 245-254.

[27]I.H. Rizvi, A. Jain, S.K. Ghosh, P.S. Mukherjee, Mathematical modelling of thermal conductivity for nanofluid considering interfacial nano-layer, Heat Mass Transfer 49 (2013) 595-600.

[28]H. Jiang, H. Li, Q. Xu, L. Shi, Effective thermal conductivity of nanofluids considering interfacial nanoshells, Material Chemistry and Physics 148(2014) 195200.

[29]H. Xie, M. Fujii, X. Zhang, Effect of interfacial nanolayer on the effective thermal conductivity of nanoparticle-fluid mixture, Int. Journal of Heat and Mass Transfer 48 (2005) 2926-2932.

[30] H.A. Mintsa, G. Roy, C.T. Nguyen, D. Doucet, New temperature dependence thermal conductivity data for water-based nanofluids, Int. Journal of Thermal Science 48(2009) 363-371.

[31] Y. Feng, B. Yu, P. Xu, M. Zou, The effective thermal conductivity of nanofluids based on the nanolayer and the aggregation of nanoparticles, Journal of Physics D: Applied Physics 40 (2007) 3164-3171. 


\section{International Journal of Science and Research (IJSR) \\ ISSN (Online): 2319-7064}

Index Copernicus Value (2013): 6.14 | Impact Factor (2015): 6.391

[32] S.M.S. Murshed, K.C. Leong, C. Yang, Investigations of thermal conductivity and viscosity of nanofluids, Int. Journal of Thermal Science 47(2008) 560-568.

[33] C. Geradi, D. Cory, J. Buongiorno, L.W. Hu, T. McKrell, Nuclear magnetic resonance-based study of ordered layering on the surface of alumina nanoparticles in water, Applied Physics Letter 95(2009)253104.

[34]E. K. Goharshadi, H. Ahmadzadeh, S. Samiee, M. Hadadian, Nanofluids for Heat Transfer Enhancement-A Review, Phys. Chem. Res., Vol. 1, No. 1, 1-33, June 2013.

[35] "An Overview of Heat Transfer Phenomena", book edited by Salim N. Kazi, ISBN 978-953-51-0827-6, and Published: October 31, 2012 under CC BY 3.0 license. (C) The Author(s). 